the masticating powers have not been destroyed from one-third to five-sixths of their capacity. We know that bolting of food day after day will produce dyspepsia, gastritis, etc., therefore we must admit that if the inability to properly masticate is not the direct cause of this dreadful disease, it certainly accompanies it. Lower your vitality and you invite disease. The oral cavity is the commencement of our digestive tract. It is the antechamber to man's vegetative life and in this respect it is introductory to the stomach. Therefore it is reasonable to suppose that any lack of hygiene, or any pathologic condition that causes a deficiency of quantity or quality of the fluids which the food first meets when entering the mouth, is a hindrance to nutrition and undoubtedly makes its impression on the stomach.

Often when a patient presents herself for treatment she is anemic, debilitated, despondent and melancholy. The physician examines her and asks various questions, but can not locate the trouble in any special organ, so he says "nervous debility," or "breaking down of the vital forces," and the usual prescription of tonics, change of residence, etc., is advised. A careful examination of the oral cavity would reveal decayed and broken down teeth and suppurating sinuses, contaminating the air of respiration, vitiating the oral fluids, poisoning nutrition and consequently lowering the vital forces and breaking down the general health. This is not imaginary, but the real condition of the mouths of many of our patients.

It is rare that we find a consumptive without a typical case of pyorrhea, or at least pus exuding from around the necks of the teeth, and I have thought that inhaling the effluvia from these suppurating sinuses of the mouth might produce this fatal disease. This leads me to ask, What should be the relations of the physician and dentist? Should the dentist know more of medicine or the physician know more of dentistry? As to these propositions I think no medical college should be without a chair on the princi. ciples and practice of dentistry and our dental schools should teach more of general medicine and surgery. 62 Inman Bldg.

\section{THE TECHNIC AND PATHOLOGY OF THE PERIDENTAL MEMBRANE.}

Read in the Section on Dental and Oral Surgery. at the Forty-seventh Annual Meeting of the American Medical Association, held at Atlanta, Ga., May 5-8, 1896.

BY VIDA A. LATHAM, M.D., D.D.S., F.R.M.S. CHICAGO, HL.

Dental histology and pathology are yet in the embryonal stage, less advanced, perhaps, than any other kindred subject. With the exception of a few, we can not claim special micro-workers. Little encouragement is given to prosecute the subject. The technic is difficult and unsatisfactory at the best, and our schools are content to teach the subject in such a brief elementary way that a student graduates in dental surgery with but a slight knowledge of the barest facts concerning the structures that compose the teeth. It is needless to urge that this is wrong, such a fact is self-evident. An exact knowledge of structure always aids in understanding the function of a part, and is absolutely essential to a clear idea of its diseases and to rational treatment. If we do not know the arrangement of enamel, we are in danger of splitting the tooth in chiseling. We can not tell what agents are absorbed if we do not know the composition of the dentinal tubules, or the action of bacteria and their septic products on the surrounding structures of the root. Precise information on the histology and chemistry of the teeth would solve many of the curious problems we meet in the practice of our profession.

So far as I can find by consulting text-books on micro-technic, little or nothing is said of technic in dentistry. A few ideas are scattered here and there through our journals. Works on dentistry dismiss in few lines the peridental membrane and its structure, position and the modes of obtaining sections for study. When looking over some mounts of teeth I was surprised at the different appearances presented, some showing extensive pathologic features and some interesting conditions. To obtain normal peridental membrane is not easy. Some authorities state that it is found on all extracted teeth, while others say it is not present unless some pathologic process exists. Certainly in the majority of healthy teeth after extraction the roots are always free from any tissue, or what might be considered periosteal tissue. In carious teeth, especially those which have had some periosteal inflammation or irritation, we can easily find thickened membrane. A recent study of several thousands of sections of carefully selected teeth has convinced me that in the case of normal teeth, after extraction, it is difficult, if not impossible, to demorsstrate the presence of peridental membrane; whereas in carious teeth, particularly those with periosteal inflammation, a thick layer of peridental membrane is always present. Therefore, I am in favor of considering sections from such teeth pathologic material.

In studying the development and relations of the peridental membrane, sections were made of teeth of animals, fetal and matured, including serial sections of one and of both jaws, to show the relations of teeth and membrane in situ. So far as I have pursued this work it has been anything but satisfactory, for the following reasons:

1. It is difficult to secure good specimens of membrane.

2. When obtained, it is hard to preserve the membrane in a normal condition and to cut sections from it.

3. Sections show poor results from staining, as the effect of some acid reagents prevents the securing of sharp definition and contrast.

4. Even with good teeth, it is difficult to secure the membrane in toto, as the paraffin sections are very liable to become brittle or curl up.

5. Much important material is lost by decalcifying agents and the irregularity of their action.

6. It is difficult to obtain maxillæ with teeth in situ which are fresh enough for examination.

Dr. Black' says: "The time the tissue remains in the embedding material must only be counted by minutes, never by hours; and if, after its removal and placing the tissue in water, it does not swell out to its normal proportions in every part, which failure may be detected after sufficient experience by its appearance, it should be cast aside and a new effort made." This statement is open to question, if we may trust our workers of technic. Poor results are too often occasioned by hurried embedding and infiltrating with the penetrating and clearing agents. If once the material has been properly and thoroughly hardened when fresh, the paraffin ought not to cause 1 Periosteum and Peridental Membrane, pp. 18 and 19. 
shrinkage. Tissues may be cut fresh in gum, ether and aniseed oil, but with these methods we expect more shrinkage. Personally, I prefer to use weak decalcifying agents for a number of days, instead of strong solutions for a short time, as advised by Dr. Black; for I believe if the material has been carefully fixed no bad shrinkage will result.

The absence of any concise discussion of this department of odontologic science in current literature is much to be deplored. So far. there is only one small compilation on the subject, and this deals chiefly with the methods for general histologic work, rather than with the methods suitable for dental microscopy. Further original investigation is much to be desired. The best way is to secure some tissue and harden portions of it in various ways, recording every particular; and then, by measuring, estimate the amount of shrinkage, the action of the hardening agents on the cells as regards the distinctness with which they show and their behavior in relation to staining agents. The previous generation of investigators have left us enormous labor in correcting their errors, for they dogmatized upon observations of tissues profoundly altered or modified in course of preparation. All the science of modern histology with its many warnings and precautions, is one great safeguard for avoiding these errors in the future. To save the tissue elements which so readily distort after death, we must secure an immediate preventive, viz., fixation. This may be done by chromic acid, Flemming's solution, Carnoy's modified by Van Hook and Ohlmacher, osmic acid, bichlorid of mercury, gold trichlorid or absolute alcohol. Fixing is especially necessary in preparing the embryonal material. When it contains cartilage, bone or dentine we may use the same fixative, but must follow it by a decalcifier. It must stay long enough in the fixing agent to harden properly and then be thoroughly washed till testing shows it acid-free. (This is not the case with certain special formulæ, which require acidity to stain well.)

The question of imbedding then follows. I obtained the best results with the freezing microtome if each section was carefully worked out. In some cases, however, it is probably necessary to use the paraffin or the celloidin method, and this particularly concerns the embryonal material. In using paraffin, even though decalcified till soft, the teeth invariably became so hard that only a few sections could be obtained from each sample. It was especially hard to avoid "crinkling" so that thin and perfectly flat sections could be made. To obtain sections of the hard and soft tissues together, the method of Drs. Lavis and Vosmaer, now better known since elaborated by Weil, is one of the best, although requiring much time and patience. Chromo-nitric or chromo-hydrochloric acids are good. Picric acid is good, but very slow in its progress. The following are a few of the most successful formulæ: Fresh teeth were dropped at once into

1. Mïller's fluid.

2. Chromic acid, 1-6 per cent., 2 parts; alcohol 1 part.-These were changed constantly, kept in the dark, then thoroughly washed and decalcified in different solutions. It was here that such contrasting results were observed.

3. Decalcification by nitric acid, C. P.-Two per cent. aqueous solution, changed daily; time required, eighteen days, to allow a needle to penetrate, then thoroughly washed and in some cases passed through an alkalin solution; then hardened in alcohol of graded strengths. Notes: "Membrane intact; pulp remained in the central canal. The membrane does not shrink and the cells stain fairly well, but not sharp and clear. Carmin gave better results than logwood. If strong solutions are used material must be closely watched, as the acid seems to act suddenly when decalcification is nearly complete, $i$. $e_{\text {., the tissues }}$ though still hard when examined may in an hour or two be all broken into useless detritus."

4. Chromic acid, 15 grs., distilled water 7 ozs., nitric acid 30 minims, changed on fourth day to one-half the strength, using fresh fluid daily. Time, eighteen days. Notes: "The membrane appears to be attacked, partially eaten off; it does not shrink; the cells stain well and the pulp is in excellent condition, showing nerves and ganglia cells in a marked manner."

5. Palladium chlorid, 0.001 per cent. in water 10 parts; hydrochloric acid 1 part. Changed once. Time, four or five days. Notes: "Does not affect the mem. brane; the results of cutting and mounting gave most perfect specimens. Staining was not sharp and clear, though somewhat improved after prolonged soaking in solutions of sodium bicarbonate or lithium carbonate and then staining for thirty-six to forty-eight hours. Care must be exercised with logwood to prevent precipitation and the stains should be renewed during the process. The pulp canal showed merely the framework, but no tissue elements. It demonstrates the longitudinal fibers nicely and stains them brown." Sections can be stained somewhat if placed in 1-300 or 1-600 solution of palladium chlorid for one to three minutes, rinsed in water and then put in a cold saturated solution of picro-carminate of soda for eight to sixty minutes. Mount in dammar. Glycerin gives good results here as a mounting medium for immediate use; but for hard or non-decalcified specimens, it affects the structure later. Equal parts of palladium and platinum chlorid is useful where a thickened membrane is found, as it shows connective tissue well.

6. Phloroglucin is a good decalcifier and preserves all elements but red blood cells. The agent itself covers the organic part of the tissue against the action of the mineral acids, which can therefore act on the inorganic structure in a more concentrated solution. It is the most rapid decalcifier I have used for bony structures. I recommend great caution in its use for teeth, which it affects somewhat more slowly. To increase its rapidity of action 35 to 45 per cent. nitric acid C. P. can be added and then wash for two days in running water. Staining is easily accomplished after using phloroglucin. If hydrochloric acid is preferred to nitric it may be added in the proportion of 30 per cent. acid with 0.5 per cent. sodium chlorid.

7. Arsenic acid, 2 per cent. aqueous solution, changed daily. Time, fifteen days. "Results were uniformly good; membrane normal, cells stain sharply, pulp shows well, filling the canal completely. Arsenic evidently has some special staining powers, as seen in the nuclei of the leucocytes."

8. Hydrochloric acid and nitric acid.-[Hopewell Smith's method. $\left.{ }^{2}\right]$ Harden three to four weeks in Müller's fluid; alcohol ten to twenty days; wash in water. Seal the apical foramen with collodion. Immerse in a 15 per cent. solution of the following to decalcify: Hydrochloric acid 12 parts, nitric acid 30 parts, aquæ dest. 108 parts. Immerse in 12 c.c. of 
a 10 per cent. solution of hydrochloric acid, then after fifteen hours add 1.5 c.c. of nitric acid, and after fortyeight hours add again 1.5 c.c. of nitric acid. After seventy-five to eighty hours wash half an hour in a solution of lithium carbonate, 5 grains to the ounce of water; wash in water, cut in pieces, place in gum mucilage and cut.

9. Hydrochloric acid and glycerin, in the proportion of hydrochloric acid 5 parts, glycerin 95 parts; is very slow in action, but softens the teeth and preserves the tissues nicely.

10. Perenyi's fluid is a most useful agent and gives good specimens, though the subsequent staining is not sharp.

11. Trichlorill of gold, 0.5 per cent. aqueous solution, is verv useful to demonstrate the transverse fibers, provided the teeth are fresh. Underwood's method may be used when the teeth are not so perfectly fresh.

Staining agents.--The stains used were logwood, borax carmin, picro-carmin, orange and rubin [S.], Weigert's method; dehydration in clove oil or cedar oil. Alum-carmin and glycerin are both liable to dissolve carbonate of lime, and should not be used to preserve pulp stones, etc.

Preservative and mounting media.-Cut sections are apt to curl up when preserved in alcohol. This may be prevented by adding a small proportion of glycerin or carbolic acid to the alcohol. If preferred, 1 or 2 per cent. of the standard 40 per cent. solution of formalin keeps indefinitely. For mounting, balsam in xylol, chloroform, glycerin and glycerin-jelly, monobromid of naphtha and dammar.

Conclusions.-1. No perfect decalcifier has been found; but arsenic acid, phloroglucin, palladium chlorid, nitric acid and chromic acid give fair results, preferred in the order named.

2. It is difficult to stain perfectly after decalcification, except in case of phloroglucin and chromic acid. If a satisfactory stain could be found to use after palladium, I would prefer the latter as a decalcifier, even though expensive, for a small quantity of the solution accomplishes much. ${ }^{3}$

808 Morse Avenue.

\section{CHRONIC RINGWORM OF THE SCALP.}

Read on Opeuing Discussion on "Chronic Ringworm of the Scalp" in n Dermatology and Syphilography, at the Fort seventh Aunual Meeting of the American Medical Association, at Atlanta, Ga., May 5-8, 1896 .

BY L. DUNCAN BULKLEY, A.M., M.D. NEW YORK.

Ringworm of the scalp has come to be regarded by those who know most about it as one of the most troublesome diseases of the skin; it is also much more frequent than is commonly supposed.

While the other forms of trichophytic disease occur more or less frequently in adults, its appearance on the scalp belongs especially to childhood. In the writer's private practice there were only 2.5 per cent. of patients thus affected who were over 13 years of age, and about the same proportion in public prac. tice; it may affect those but a few months old.

The damage that the parasite may do in some patients is so slight that the disease is often overlooked and undoubtedly there are many cases which

3 See Histology of the Teeth, Notes on Preparation, ete.; Histology of the Teth, Internatonal J. of Microscopy and Natural Science. 1889, July and October $1892 ; 189$, , January and October; Histology of the Teeth, go for years untreated, endangering those around them, as the writer has repeatedly observed both in public and private practice. Alder Smith, of London, ${ }^{1}$ has known an outbreak of ringworm to occur in a school from a child with a single spot no larger than a split pea, and where only a few "stumps" could be found on close examination with a lens. Among 1,349 healthy boys, aged from 8 to 10 years, whom he examined for admission to a charity school in London, and who were supposed to be quite free from ringworm of the head, he found this present in 112 cases, or 8.3 per cent. of the whole.

Many cases which came to him with certificates from reputable physicians that their ringworm was cured, were found on careful study to have abundance of the disease in a chronic disseminated form.

In one public institution in New York City, shel. tering between seven and eight hundred children, 367 cases of ringworm of the scalp were found, that is, over half of the inmates were infected before the damage was fully realized and active measures for its arrest were inaugurated.

Tinea tonsurans of the scalp is not always as easy of recognition as might be supposed, and in cases which have lasted for any length of time the diagnosis may be very difficult. The ty pical case of scalp ringworm, exhibiting one or more partially bald patches, of a more or less leaden color, and with stubbed hairs, may be recognized by everyone. But in cases which have lasted any length of time the affected surfaces may and generally do not present this typical tonsure appearance. After a certain duration, and especially after more or less satisfactory treatment, many of the hairs assert their vigor (although by the microscope they may present some evidence of the parasite) and will grow to a considerable length. The real nature of the disease is thus masked and it is only by the most careful search that the "stump" or broken-off hairs can be detected.

To this persistent and rebellious form of the disease the name of chronic or disseminated ringworm has been given, and it is one of the most troublesome affections which can be presented for treatment.

Few realize how exceedingly persistent ringworm of the scalp may become in certain cases. The disease is commonly looked upon as a trivial matter, and undoubtedly in healthy children many cases may yield quite promptly to relatively simple treatment; even tincture of iodin will cure, or seem to cure, a good number of cases. But on the other hand, quite a number of these patients who are supposed to be cured are afterward found still to present evidence of the parasite, which crops out afresh later and, as already mentioned, may start the disease in a school or family.

The physician should therefore be extremely careful whenever scaly conditions of the scalp are presented in children, to determine with positiveness if the trichophyton is present. When the parasite exists on the scalp or hairs, the greatest care should be exercised to prevent the disease spreading to others. And when the patient has been under treatment for this eruption, still greater care should be exercised, if possible, in deciding as to when the patient is cured and no longer capable of infecting others. Few cases of ringworm of the scalp can be thoroughly cured in a short time, such as a month; more frequently from

1 Alder Smith: Ringworm Dingnosis and Treatment. 2d edition. London. 